\title{
GENERATION OF AN OPTIMAL LOW-ALTITUDE TRAJECTORY FOR A FIXED-WING UNMANNED AERIAL VEHICLE IN A MOUNTAINOUS AREA
}

\author{
Hossein MAGHSOUDI, Amirreza KOSARI* \\ Aerospace Department, Faculty of New Sciences and Technologies, University of Tehran, Tehran, Iran
}

Received 04 December 2019; accepted 05 June 2020

\begin{abstract}
In this study, the three-dimensional optimal trajectory planning of an unmanned fixed-wing aerial vehicle was investigated for Terrain Following - Terrain Avoidance (TF-TA) purposes using the Direct Collocation method. For this purpose, firstly, the appropriate equations representing the translational movement of the aircraft were described. The three-dimensional optimal trajectory planning of the flying vehicle was formulated in the TF-TA manoeuvre as an optimal control problem. The terrain profile, as the main allowable height constraint was modelled using the Fractal Generation Method. The resulting optimal control problem was discretized by applying the Direct Collocation numerical technique and then, was transformed into a Nonlinear Programming Problem (NLP). The efficacy of the proposed method was demonstrated by extensive simulations, and it was particularly verified that the purposed approach can produce a solution satisfying almost all the performance and environmental constraints encountering in a low -altitude flight.
\end{abstract}

Keywords: trajectory planning, Terrain Following - Terrain Avoidance (TF-TA), unmanned fixed-wing aerial vehicle, Direct Collocation method.

\section{Introduction}

Over the past three decades, Unmanned Aerial Vehicles (UAVs) have been well considered due to their numerous merits such as low manufacturing cost, advanced automatic control systems, withstanding the high accelerations, and high maneuverability. Since these robots are widely employed for low-altitude mission purposes, such as traffic control therefore, they are significantly considered nowadays. On the other hand, Terrain Following and Terrain Avoidance (TF-TA) are among the main challenges discussed in terms of low-altitude flight, especially, in the mountainous areas (Kassaei \& Kosari, 2018). Since, nowadays, many airports are located in the mountainous areas with adverse weather conditions; probability of the accidents occurring near these airports will also be increased (Pourtakdoust et al., 2011). According to the above-mentioned issues, obviously, safe landing conditions are very difficult for a UAV with the TF-TA capability (Babaei \& Karimi, 2018). Therefore, some approaches should be considered for solving the problem based on the missions defined for a UAV (Kosari et al., 2017).

Generally, the Terrain Following problems could be classified into two categories based on their path planning and guidance (Jalali-Naini \& Ebrahimi, 2017; Jalali-Naini \& Sajjadi, 2016). Considering a two-dimensional motion for the flying vehicle in vertical plane is the most common guidance method and, as a result, only altitude variations in the flight plane are taken into consideration. TF is generally germane to the above-mentioned problems. Another problem considering the motion, in the horizontal plane is known as Terrain Avoidance (TA) (Kosari et al., 2015). For comparing the TF and TA maneuvers, it could be mentioned that, angle of attack and velocity alter in TF, whereas in TA, flying vehicle could pass the terrain during its lateral maneuvers with respect to the velocity variations and heading angle. The methods considering the three-dimensional motion of the flying vehicle are various combinations of the TF and TA maneuvers, which are exclusively regarded as the TF-TA (Bagherian, 2018; Kamyar \& Taheri, 2014; Malaek \& Kosari, 2012).

One of the preponderant scopes in the TF-TA maneuver is finding an optimal trajectory for a flying vehicle in such a way that a set of the constraints, such as initial and final conditions, dynamic constraints, and so forth are satisfied in the best possible fashion (Malaek \& Kosari, 2007). In fact, all of the UAVs flying in this condition require a safe distance from the terrain to increase the flight endurance

*Corresponding author. E-mail: kosari_a@ut.ac.ir 
and mission effectiveness. Generally, it is desired that the flying robot passes the afore-mentioned areas by maximum possible velocity (minimum time) and minimum trajectory (the minimum length of path or minimum fuel) so that, its altitude could be decreased as far as possible. In fact, there is always a tradeoff between designers' requirements and peculiar conditions in the flight areas (Kazemifar et al., 2017; Babaei \& Mortazavi, 2010). Generally, the scope of all the issues related to the TF maneuver for UAVs includes (Malaek \& Kosari, 2012):

1. Trajectory generation with TF purposes to enhance both survivability and mission effectiveness;

2. Development of controlling laws to follow the actual trajectory or model it regarding the maneuvering capabilities of the flying vehicle;

3. Integration of the sensors for updating the terrain data;

4. Evaluation of the integrated system for different scenarios as well as emergencies.

In other words, path planning problem is defined for generating a desired trajectory with the purposes including the minimum length of path, minimum time, collision avoidance, minimum control effort, minimum fuel, and so forth, based on the missions of the aircrafts. In some researches on the trajectory generation, geometric constraints are applied for developing the optimization problems. In these researches, dynamic features of the flying vehicles such as rotational velocity and maximum allowable bank angle are converted into the equivalent geometric constraints. Therefore, the main obligation of the designers is finding an optimal trajectory so as to meet the imposed geometric constraints. As mentioned earlier, the main objective of this research is three-dimensional optimal path planning of a flying vehicle using the Direct Collocation method (Hargraves \& Paris, 1987), therefore, the dynamic/kinematic constraints are directly imposed into the problem's formulation without any need to convert into geometric constraints (Grimm \& Hiltmann, 1987; Von Stryk \& Bulirsch, 1992). The outstanding privilege of this work is that, in some cases, there are specific dynamic constraints, which cannot be converted into geometric constraints easily, therefore, this challenge is completely solved in this research. In fact, one of the main objectives of this study is simplifying the TF-TA flight mode in such a way that generated trajectory could be utilized for real time applications as well as the terrain versatility.

Generally, solving methods for the trajectory optimization problems could be divided into two classes as follows (Betts, 2010; Conway, 2012; Huang et al., 2012; Rao, 2009):

1. Direct methods;

2. Indirect methods.

The indirect methods are based on calculation of variations in such a way that the trajectory optimization problem is transformed into a Hamiltonian Boundary Condition Problem where this transformed problem should be solved using the numerical methods, whereas in the direct methods, the trajectory optimization problem changes into a Nonlinear Programming Problem (NLP) with bounded dimensions (Betts, 1998). In the indirect methods, first, some extra variables such as co-state variables are obtained and then, control and state variables are calculated by utilizing those variables, whereas in the direct methods, the control and state variables could be obtained, directly without using the extra variables. Notwithstanding the numerous merits, there are some demerits in the indirect methods as noted below (Betts, 1998; Garg, 2011; Benson, 1978; Fahroo \& Ross, 2008):

1. In these methods, optimality conditions are analytically required and, as a result, this issue is time consuming in some cases.

2. These methods require initial guess for auxiliary variables. Therefore, since there is no physical sense towards these variables, solving the problem is very difficult.

3 . These methods have very low convergence amplitude, so proposed answers should be close to the real answers so that, the answers converge. Therefore, the solving speed is very low.

Direct methods are widely proposed instead of indirect ones on account of solving the aforementioned drawbacks. Direct methods have high convergence relative to the indirect ones. Moreover, they do not require the costate variables' guess and also they are not sensitive to the initial guess. It should be noted that, the direct methods have also some demerits. For instance, optimal control problem is converted into a large-scale optimal problem through these methods. On the one hand, the number of nodes should be high for obtaining a desired accuracy, which increases the NLP dimension.

It should be mentioned that each aforementioned method could be classified in either direct or indirect categories. For instance, the inverse dynamics and steepest descent approaches belong to the indirect methods and the Direct Collocation and pseudo-spectral approaches are classified as the direct methods. As mentioned earlier, the main objective of this research is generating the three-dimensional optimal trajectory using the Direct Collocation in such a way that TF-TA constraints and all the requirements are satisfied.

The outline of the paper is as follows. In Section 1, equations of motion for the flying robots are presented. The TF problem is formulated in Section 2. Section 3 is dedicated to the Direct Collocation approach for generating the three-dimensional optimal trajectories. Simulation results are presented in Section 4, and finally the conclusions of this research have been discussed.

\section{Equations of motion for the flying vehicle}

The equations representing the translational motion of a fixed-wing UAV in three-dimensional space should be established for generating the optimal trajectory. The system equations for optimal control problem are generally presented as follows (Sharma et al., 2005; Von Stryk \& Bulirsch, 1992):

$$
\frac{d x}{d t}=f(x, u, t) \text {, }
$$


where, $x$ is a $n \times 1$ state vector, $u$ is a $m \times 1$ ontrol vector, $t$ stands for time, and $f$ is a given function. The dynamic equations of motion for a flying vehicle in three-dimensional space with a flat ground, standard atmosphere, and under the assumption of a point mass are defined as (Sharma, 2006; Sharma et al., 2005):

$$
\begin{aligned}
& \frac{d x}{d t}=V \cos \gamma \cos \psi ; \\
& \frac{d y}{d t}=V \cos \gamma \sin \psi ; \\
& \frac{d z}{d t}=V \sin \gamma ; \\
& \frac{d V}{d t}=\frac{1}{m}[T \cos \alpha-D]-g \sin \gamma ; \\
& \frac{d \psi}{d t}=\frac{[T \sin \alpha+L] \sin \phi}{m V \cos \gamma} ; \\
& \frac{d \gamma}{d t}=\frac{[T \sin \alpha+L] \cos \phi}{m V}-\frac{g}{V} \cos \gamma,
\end{aligned}
$$

where, in Equations $(2-4),(x, y, z)$ indicate the coordinates of the flying vehicle in three-dimensional space, $V$ is the velocity of the flying vehicle, $\gamma$ is the flight path angle, and $\psi$ is heading angle, which are defined as state variables. Moreover, in Equations (5-7), $\alpha, \phi$, and $m$ are angle of attack, rolling angle, and aircraft mass, respectively.

Furthermore, in Equations (5-7), $L$ and $D$ represent the lift and drag forces, respectively, which are noted below:

$$
\left\{\begin{array}{l}
L=q S C_{L} \\
D=q S C_{D}
\end{array}\right.
$$

where, $q$ is the dynamic pressure and $S$ is the reference wing area of the flying vehicle which itself is the function of air density $(\rho)$ and velocity. $C_{L}$ and $C_{D}$ are the lift and drag coefficients, which are the functions of angle of attack $(\alpha)$ and Mach number $(M)$. The propulsion force is defined as:

$$
T=T_{\max }(M, z) \Gamma(\eta),
$$

where, $\Gamma(\eta)$ denotes the engine power introduced as throttle and alters in the $0 \leq \eta \leq 1$ range. Whenever, $\eta$ is zero, the engine is off, and whenever $\eta$ is one, the engine has the maximum thrust force. Furthermore, the symbol $\Gamma$ represents a nonlinear function of $\eta$. In Equation (9), $T_{\max }$ indicates the maximum thrust force for $\eta=1$.

Some of the dynamic physical features related to the discussed flying robot are presented as follows (Malaek \& Kosari, 2007):

$$
\begin{aligned}
& C_{L}=\alpha C_{L_{\alpha}} ; \\
& C_{D}=C_{D_{0}}+K_{L}{ }^{2} .
\end{aligned}
$$

In Equations 10-11, $\mathrm{C}_{\mathrm{L} \alpha}$ is a function of Mach number $\mathrm{M}$, while $\mathrm{CD}_{0}$ is a function of Mach number and flying altitude $\mathrm{z} . \mathrm{C}_{\mathrm{L} \alpha}$ and $\mathrm{C}_{\mathrm{D} 0}$ are provided in a tabulated form (Malaek \& Kosari, 2007). In this paper, Least Squares method is also utilized to generate continuous functions for both $\mathrm{C}_{\mathrm{L} \alpha}$ and $\mathrm{C}_{\mathrm{D} 0}$ in terms of Mach number for a specified aircraft (See Malaek \& Kosari, 2007; Kosari \& Kassaei, 2019). The suitable range for $M$ was found to be from 0.0 to 0.9 . These functions are given by the Equations (12-13).

$$
\begin{aligned}
& C_{L_{\alpha}}=4.198-0.3425 \times \mathrm{M}+1.0125 \times M^{2} ; \\
& \log \left(C_{D_{o}}\right)=-2.02037-0.078043 \log (M)+ \\
& 0.05707 \log (z) .
\end{aligned}
$$

The mathematical model of the aircraft propulsion force only representing the static model is presented as follows (Malaek \& Kosari, 2007):

$$
\mathrm{T}=\left\{\begin{array}{lr}
\left(\eta^{6}+N(\mathrm{M}+z)\right) \mathrm{T}_{\max }(\mathrm{M}, z) & \text { if } \eta \neq 0 \\
0 & \text { if } \eta=0
\end{array} .\right.
$$

In this research, there are three control variables selected based on their equations of motion. These control variables are angle of attack, rolling angle, and throttle position, which are shown by $\alpha, \phi$, and $\eta$, respectively. It should be mentioned that, the defined state and control variables in this problem are bounded, that is, they could be altered in a specific range, which has been already defined. This range depends upon the aircraft dynamics and its performance. The below constraints were defined for control variables as amplitude variations:

$$
\begin{aligned}
& \alpha_{\min } \leq \alpha \leq \alpha_{\max } \\
& \varphi_{\min } \leq \varphi \leq \varphi_{\max } \\
& 0 \leq \eta \leq 1
\end{aligned}
$$

In the next section, TF formulation is presented in great details.

\section{TF Formulation}

Formulation of the optimal control problem usually requires some factors as follows:

1) A mathematical model of the system dynamics, which should be controlled;

2) A defined cost function;

3) All the constraints including initial and terminal boundary conditions, path constraint, and so forth.

The objective function for each optimal control problem is generally defined as:

$$
J=\Phi\left(x_{f}, t_{f}\right)+\int_{t_{0}}^{t_{f}} \mu(x, u, t) d t,
$$

where, $\mu$ and $\Phi$ are nonnegative functions. In this research, the defined mission is passing the trajectory above the terrain in such a way that the flying vehicle reaches to the specific destination in either shortest possible time or minimum length of path. According to this issue, the minimum length of path cost function is defined in this problem, as follows (Kosari, \& Kassaei, 2019):

$$
J=\int_{t_{0}}^{t_{f}} \sqrt{\left(\frac{d x}{d t}\right)^{2}+\left(\frac{d y}{d t}\right)^{2}+\left(\frac{d z}{d t}\right)^{2}} d t
$$


Formulation of the TF-TA manoeuvres is similar to the two-point boundary value problem, therefore the optimal trajectory should satisfy the flight initial and terminal conditions. The constraints in initial and terminal points are generally classified into equality and inequality constraints. In this research, the initial and terminal conditions are only considered as equality constraints defined as:

$$
\begin{aligned}
& \Psi\left(x_{0}, t_{0}\right)=0 ; \\
& \Psi\left(x_{f}, t_{f}\right)=0,
\end{aligned}
$$

where, $t_{0}$ is the initial time, $x_{0}$ is the state vector at the initial time, $t_{f}$ is the final time (that can be assumed to be fixed or free), $x_{f}$ is the state vector at the final time, and $\Psi$ is a vector consisting of $R \times 1$ functions of the state variables, initial , and final time.

In this research, the initial time was specified, whereas the final time was considered as unspecified based on either the designers' requirements or the mission type. Since, the problem is a two-point boundary value problem, dynamic equations of the flying vehicle should be formulated in terms of another variable such as $x, y$, or $z$. Therefore, there is no dependency upon time because initial $\left(x_{i}, y_{i}, z_{i}\right)$ and final $\left(x_{f}, y_{f}, z_{f}\right)$ positions are specified in this problem. In this research, the equations presenting the motion of the flying vehicle were formulated in terms of the $x$ so, they were converted into new forms as follows:

$$
\begin{aligned}
& \frac{d y}{d x}=\tan \psi ; \\
& \frac{d z}{d x}=\frac{\tan \gamma}{\cos \psi} ; \\
& \frac{d V}{d x}=\frac{T \cos \alpha-D}{m V \cos \gamma \cos \psi}-\frac{g}{V} \times \frac{\tan \gamma}{\cos \psi} ; \\
& \frac{d \psi}{d x}=\frac{[T \sin \alpha+L] \sin \phi}{m V^{2} \cos ^{2} \gamma \cos \psi} ; \\
& \frac{d \gamma}{d x}=\frac{[T \sin \alpha+L] \cos \phi}{m V^{2} \cos \gamma \cos \psi}-\frac{g}{V^{2}} \times \frac{1}{\cos \psi} .
\end{aligned}
$$

On the one hand, new form of the objective function is noted below:

$$
\begin{aligned}
& J=\int_{t_{0}}^{t_{f}} \sqrt{\left(\frac{d x}{d t}\right)^{2}+\left(\frac{d y}{d t}\right)^{2}+\left(\frac{d z}{d t}\right)^{2}} d t=\int_{x_{0}}^{x_{f}} A d x ; \\
& A=\sqrt{1+(\tan \psi)^{2}+\left(\frac{\tan \gamma}{\cos \psi}\right)^{2}}= \\
& \sqrt{\frac{\cos \psi^{2}}{\cos \psi^{2}}+\frac{\sin \psi^{2}}{\cos \psi^{2}}+\frac{\tan \gamma^{2}}{\cos \psi^{2}}}=\sec \gamma \times \sec \psi .
\end{aligned}
$$

There are also some environmental constraints in the path planning problems, such as obstacles avoidance constraints, box constraints over the state and control variables. These constraints were classified into two categories of equality and inequality constraints, as noted below:
Equality constraints:

$$
c_{e q}(x, u, t)=0 \text {. }
$$

Inequality constraints:

$$
c_{\text {ineq }}(x, u, t) \leq 0 \text {, }
$$

Where, $c_{e q}$ is a $q \times 1$ vector and $c_{\text {ineq }}$ is a $p \times 1$ vector. In this study, external constraints are altitude constraints in terrain form so that, the flying robots can keep a prespecified distance from the terrain and obstacles for the purpose of collision avoidance. These constraints are imposed as follows:

$$
\begin{aligned}
& \operatorname{Terrain}(x, y)+h_{\min } \leq z(x, y) \\
& \operatorname{Terrain}(x, y)+h_{\max } \geq z(x, y) .
\end{aligned}
$$

\section{Direct Collocation approach}

The main idea underlying the Direct Collocation method is discretizing the state and control variables through either linear or higher -order interpolation such as Euler method, Trapezoidal method, Hermite-Simpson method, and Classical Runge-Kutta method. Therefore, in the Direct Collocation method, an optimal control problem is discretized in such a way that differential and integral equations are converted into algebraic equations. In other words, an optimal control problem changes into a standard algebraic equation in the form of NLP using this method. Time interval $\left[t_{i}, t_{f}\right]$ in this method is divided into several small intervals. As shown in Figure 1 , each time interval is between two nodes and these intervals could be considered either the same or different. It could be mentioned that, herein, the intervals were selected the same for further simplicity. If the number of nodes and time interval are regarded as $n$ and $h_{i}$ respectively, so:

$$
h_{i}=\frac{t_{f}-t_{i}}{n-1} .
$$

An optimal control problem with two state variables $(x, y)$ and control variable $(u)$ will be as follows:

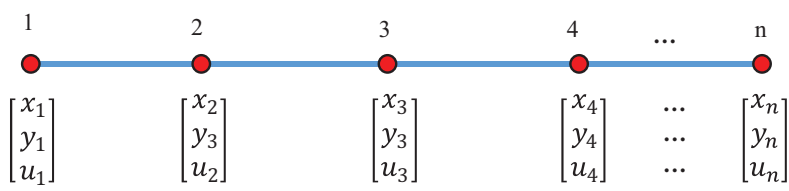

Figure 1. Schematic of the discretized trajectory with state and control variables

State and control values should be determined in each node to solve the optimal control problem using the Direct Collocation method. For this purpose, each of the state and control values in each node is regarded as a variable. Accordingly, all the state and control variables could be put in the same matrix and regarded as the same variables like variable a, as noted below: 


$$
\left[\begin{array}{cccc}
x_{1} & x_{2} & \cdots & x_{n} \\
y_{1} & y_{2} & \cdots & y_{n} \\
u_{1} & u_{2} & \cdots & u_{n}
\end{array}\right]=\left[\begin{array}{cccc}
a_{1} & a_{2} & \cdots & a_{n} \\
a_{n+1} & a_{n+2} & \cdots & a_{2 n} \\
a_{2 n+1} & a_{2 n+2} & \cdots & a_{3 n}
\end{array}\right] .
$$

Therefore, the values of $a_{i}$ should be obtained for solving the optimal control problem. Now, the state equations are required to be presented based on the $a_{i}$ variables. Suppose that, the state equations are generally described as follows:

$$
\frac{d x}{d t}=f(x, u)
$$

As already noted, there are numerous methods for discretizing the aforementioned equation two of which are illustrated in great detail.

\subsection{Trapezoidal method}

The above-mentioned equation could be discretized through the Trapezoidal approximation as noted below:

$$
\frac{d x}{d t}=\frac{x_{i+1}-x_{i}}{h_{i}}=\frac{f\left(x_{i}, u_{i}\right)+f\left(x_{i+1}, u_{i+1}\right)}{2} \text {. }
$$

Moreover, the equation's estimation between two nodes should be satisfied, therefore the aforementioned equation could be regarded as an equality constraints in such a way that error vector between two nodes becomes zero:

$$
d_{i}=x_{i+1}-x_{i}-\frac{h_{i}}{2} \times\left[f\left(x_{i}, u_{i}\right)+f\left(x_{i+1}, u_{i+1}\right)\right]=0 \text {. }
$$

\subsection{Hermite-Simpson method}

If Simpsons' integration rule and Hermites' interpolation method are used instead of Trapezoidal integration rule, the error vector for each node is calculated as follows:

$$
d_{i}=x_{i+1}-x_{i}-\frac{h_{i}}{2}\left[f\left(x_{i}, u_{i}\right)+f\left(x_{i+1}, u_{i+1}\right)\right] \text {, }
$$

where, $x_{c i}, u_{c i}$ are the state and control variables vector in the centres of the $i$-th and $(i+1)$-th nodes, respectively, and are defined as:

$$
\begin{aligned}
& x_{c i}=\frac{1}{2}\left(x_{i+1}-x_{i}\right)-\frac{h_{i}}{8}\left[f\left(x_{i}, u_{i}\right)-f\left(x_{i+1}, u_{i+1}\right)\right] ; \\
& u_{c i}=\frac{1}{2}\left(u_{i}+u_{i+1}\right) .
\end{aligned}
$$

In the above relations, $i$ could change from 1 to $n$, and the vector $d_{i}$ is referred to as the error in node $i$. If the state and control vectors in the nodal points are selected in such a way that the values of error vector are equal to 0 for $I=1,2, \ldots, n$, the differential equations of motion are satisfied in a discrete form.

As mentioned earlier, two methods including Trapezoidal and Hermite-Simpson methods were applied in this research for discretizing the differential equations of the flying vehicle motion and converting them into a NLP. Simulation results based on two different models of the terrain and using two aforementioned methods are presented in the next section.

\section{Simulation results}

As mentioned frequently, the main objective of this research is generation of the three-dimensional optimal trajectory using the Direct Collocation method for the TFTA purposes. Therefore, simulation results are represented for a model of the terrain through two discretizing methods including Trapezoidal and Hermite-Simpson in this section. Tables 1 and 2 present the physical constraints of the aircraft and boundary conditions as well as constraints of the state variables obtained based on modeling the fractal terrain generation, respectively. Moreover, simulation results based on the aforementioned terrain modeling through two discretizing methods including Trapezoidal and Hermite-Simpson are shown in Figures 2-10.

Table 1. Some specifications and performance constraints of the UAV

\begin{tabular}{|l|l|}
\hline \multicolumn{1}{|c|}{ Physical parameters } & \multicolumn{1}{c|}{ Value } \\
\hline Reference wing area of the UAV & $S=2.67\left(\mathrm{~m}^{2}\right)$ \\
\hline Primary mass of the UAV & $m_{0}=250(\mathrm{~kg})$ \\
\hline Minimum angle of attack & $\alpha_{\min }=-5^{\circ}$ \\
\hline Maximum angle of attack & $\alpha_{\max }=8^{\circ}$ \\
\hline Minimum flight path angle & $\gamma_{\min }=-12^{\circ}$ \\
\hline Maximum flight path angle & $\gamma_{\max }=12^{\circ}$ \\
\hline Minimum rolling angle & $\varphi_{\min }=-25^{\circ}$ \\
\hline Maximum rolling angle & $\varphi_{\max }=25^{\circ}$ \\
\hline $\begin{array}{l}\text { Minimum allowable altitude from the } \\
\text { ground }\end{array}$ & $h_{\min }=10(\mathrm{~m})$ \\
\hline $\begin{array}{l}\text { Maximum allowable altitude from the } \\
\text { ground }\end{array}$ & $h_{\max }=350(\mathrm{~m})$ \\
\hline
\end{tabular}

Table 2. Boundary conditions and constraints of the state variables obtained based on modeling the fractal terrain generation

\begin{tabular}{|l|l|}
\hline \multicolumn{1}{|c|}{ Constraints } & \multicolumn{1}{|c|}{ Value } \\
\hline $\begin{array}{l}\text { The initial position of the UAV alongside } \\
\text { the longitudinal axis }\end{array}$ & $x_{i}=1500(\mathrm{~m})$ \\
\hline $\begin{array}{l}\text { The final position of the UAV alongside the } \\
\text { longitudinal axis }\end{array}$ & $x_{f}=9050(\mathrm{~m})$ \\
\hline $\begin{array}{l}\text { The initial position of the UAV alongside } \\
\text { the lateral axis }\end{array}$ & $y_{i}=1600(\mathrm{~m})$ \\
\hline $\begin{array}{l}\text { The final position of the UAV alongside the } \\
\text { lateral axis }\end{array}$ & $y_{f}=570(\mathrm{~m})$ \\
\hline $\begin{array}{l}\text { The initial position of the UAV alongside } \\
\text { the altitude }\end{array}$ & $z_{i}=14.5(\mathrm{~m})$ \\
\hline $\begin{array}{l}\text { The final position of the UAV alongside the } \\
\text { altitude }\end{array}$ & $z_{f}=410(\mathrm{~m})$ \\
\hline Minimum velocity of the UAV & $V_{\min }=42(\mathrm{~m} / \mathrm{s})$ \\
\hline Maximum velocity of the UAV & $V_{\max }=80(\mathrm{~m} / \mathrm{s})$ \\
\hline
\end{tabular}




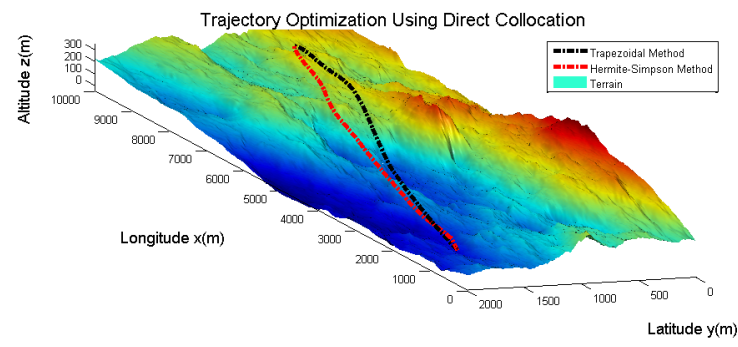

Figure 2. Schematic of the three-dimensional trajectory with the terrain

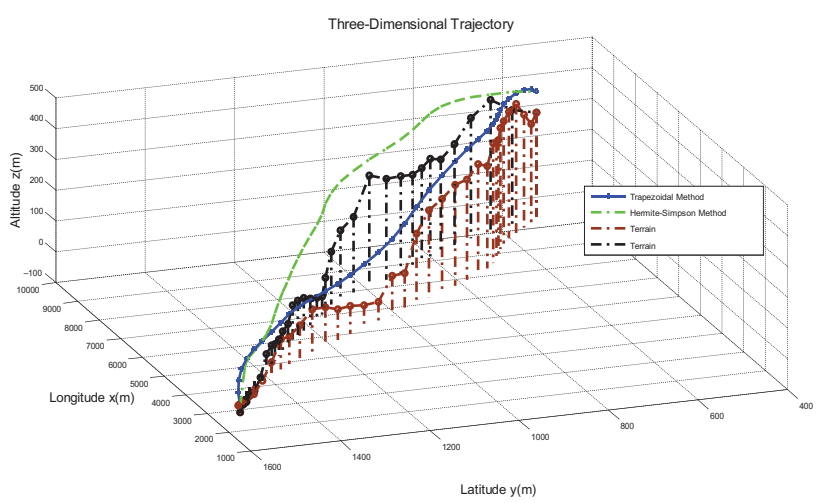

Figure 3. Simplified view of the terrain with trajectory

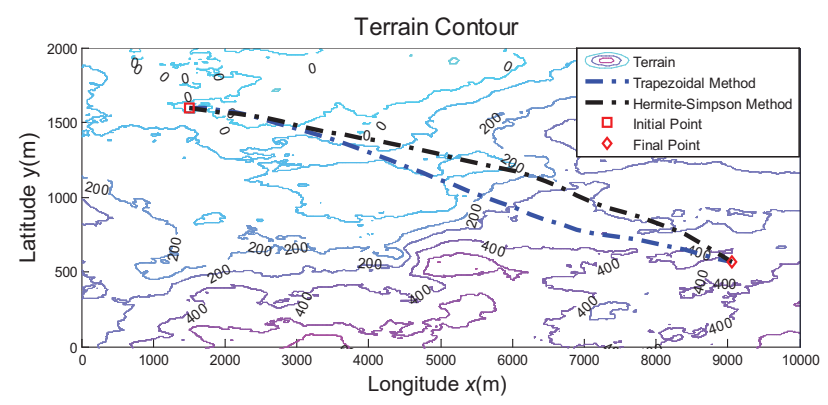

Figure 4. Optimal trajectory with the terrain contour

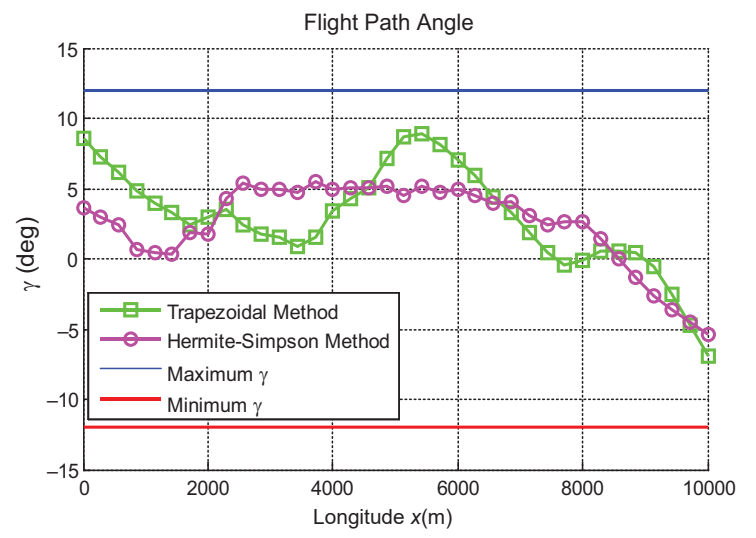

Figure 5. Curve of the flight path angle

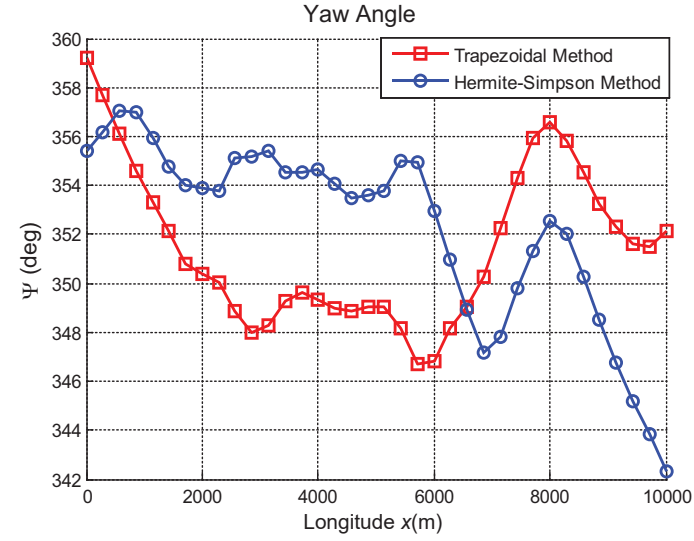

Figure 6. Yaw angle curve

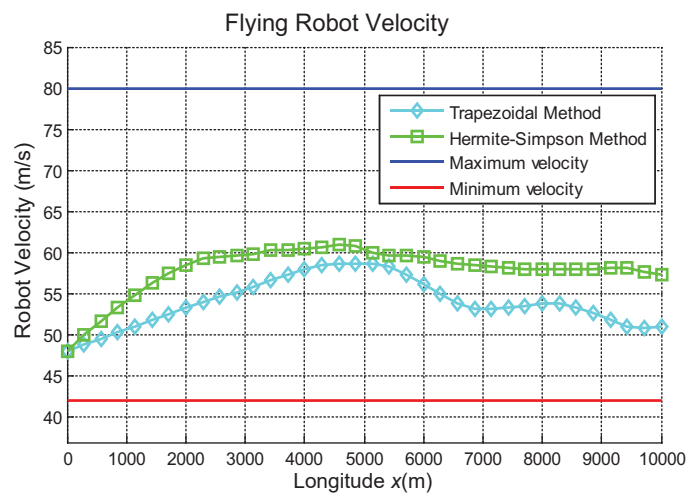

Figure 7. Velocity curve of the UAV

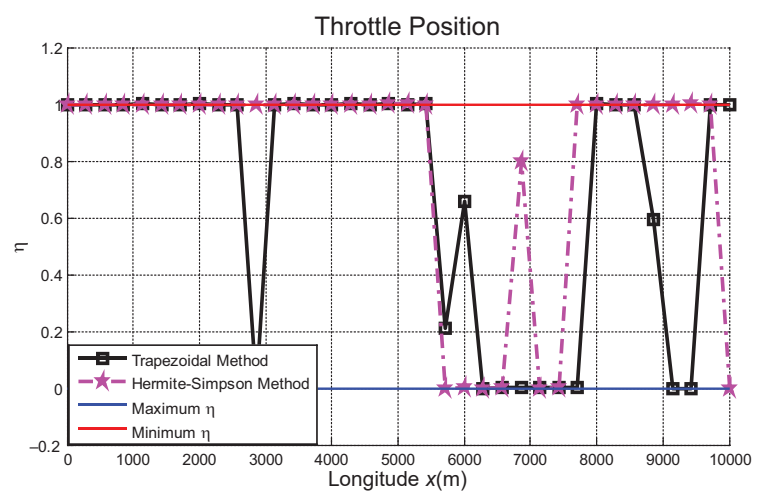

Figure 8. History of the throttle controller

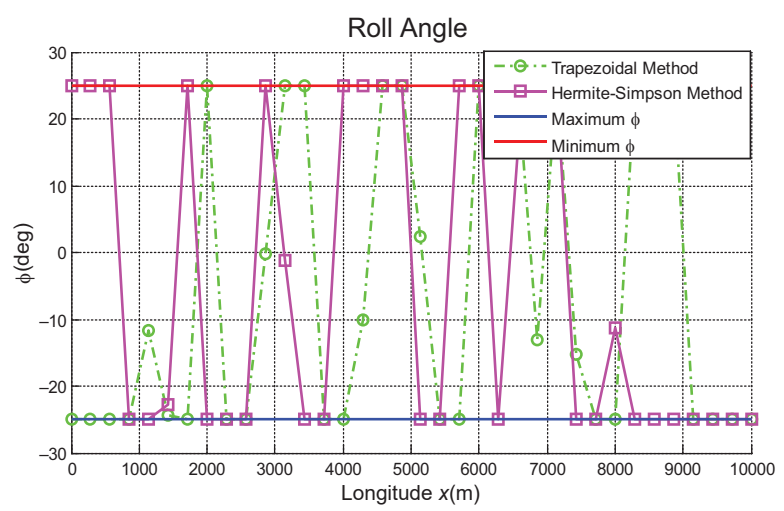

Figure 9. History of the roll angle controller 


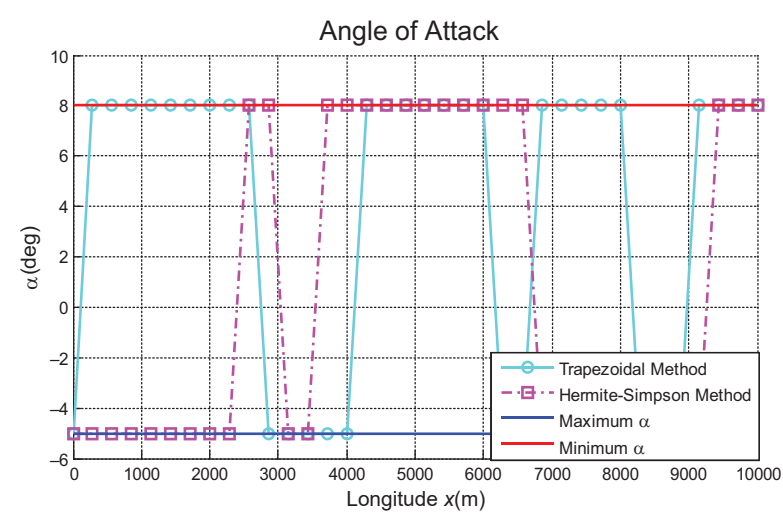

Figure 10. History of the angle of attack controller

The objective of this paper is to obtain an optimal reliable path design approach to control a flying robot in the vicinity of the ground similar to TF flights and be the minimum length. As shown in Figures 2-10, simulations demonstrate that the obtained trajectory is near the terrain and as much as possible and meets all of the constraints.

\section{Conclusions}

The present study was conducted to generate the three-dimensional optimal trajectory using the Direct Collocation approach in such a way that a set of dynamic/kinematic constraints such as flight bound, terrain and the initial/ terminal boundary conditions are satisfied. First and foremost, equations of motion were presented for the UAV based on its dynamics. Then, TF problem was formulated as an optimal control problem, where the objective function was regarded as the minimum length of path. There are numerous methods for discretizing the equations two of which including Trapezoidal and HermiteSimpson methods were employed in this study for solving the problem and the results were presented based on the minimum length of path cost function. In trajectory planning, the performance index is generally formed taking into account mission requirements, functional capabilities and problem limitations, and it could be a single or multi-objective function. In most of trajectory planning problems, the objective function(s) is/are taken to be minimum control effort, shortest flight time/path, minimum fuel consumption, etc. In the current study, it was tried to obtain the path meeting all the dynamical/functional constraints and the flying corridor. Minimization of route length was also considered that was associated with the power consumption. The criterion function of minimum route length was developed given that the problem is formulated based on the position.

Firstly, equations of motion were described for the flying vehicle in the three-dimensional space with a flat ground, standard atmosphere, and under the assumption of a point mass. Then, the TF-TA problem was formulated as an optimal control problem. Since the main objective of this paper was finding the three-dimensional optimal trajectory for the TF-TA maneuvers, modeling the threedimensional terrain was one of the big challenges in this problem.

After problem formulation as an optimal control problem, Direct Collocation method was applied for solving the problem. One of the most outstanding features of this method is that the differential equations could be converted into algebraic equations through discretizing. Herein, it was verified that the proposed feasible trajectory meets all the requirements after employing the method and solving the NLP. In other words, although the dynamic equations of the aircraft were highly nonlinear, the simulation results were very promising.

Different numerical integration schemes can be employed such as Euler, Hermite-Simpson, Runge-Kutta, Runge-Kutta-Fehlberg, etc. to numerically solve an equation of motion for the UAV (Lindfield \& Penny, 2018). Each of these methods has its particular advantages and drawbacks. Schemes like Euler have low computational effort and thus acceptable solving speed, but in some cases, they lack sufficient accuracy for the integration. That might be due to large difference in the magnitude order of some variables involved in the equation (for example, $10 \mathrm{e} 6$ in comparison with 10e-3). In these cases, the state equations should be rewritten employing dimensionless variables or higher-order integration methods can be employed.

In this paper, the problem was solved using both Trapezoidal and Hermite-Simpson methods. Although, utilizing higher-order schemes such as Runge-Kutta can improve the accuracy and reliability but they may significantly increase the computational cost. According to the simulation results, Euler and Hermite-Simpson methods could effectively meet the problem requirements with acceptable accuracy.

Finally, it should be mentioned that, there is a great number of heuristic methods for solving the problems of three-dimensional optimal trajectory generation, but most of them are not capable of solving this complex problem under tight constraints. The efficacy of Direct Collocation method was elucidated in this study and it was demonstrated that the problem can be solved with a high accuracy. Therefore, this approach could be utilized in various problems of three-dimensional optimal path planning and this research could pave the way for further researches in the future.

\section{References}

Babaei, A. R., \& Mortazavi, M. (2010). Three-dimensional curvature-constrained trajectory planning based on in-flight waypoints. Journal of Aircraft, 47(4), 1391-1398. https://doi.org/10.2514/1.47711

Babaei, A., \& Karimi, A. (2018). Optimal trajectory-planning of UAVs via B-splines and disjunctive programming. In ArXiv preprint arXiv:1807.02931 (pp. 1-12). 
Bagherian, M. (2018). Unmanned Aerial Vehicle Terrain Following/Terrain Avoidance/Threat Avoidance trajectory planning using fuzzy logic. Journal of Intelligent \& Fuzzy Systems, 34(3), 1791-1799. https://doi.org/10.3233/JIFS-161977

Benson, D. (1978). A Gauss pseudo-spectral transcription for optimal control. Massachusetts Institute of Technology (pp. 1-224). Department of Aeronautics and Astronautics. http://hdl.handle.net/1721.1/28919

Betts, J. T. (1998). Survey of numerical methods for trajectory optimization. Journal of Guidance, Control, and Dynamics, 21(2), 193-207. https://doi.org/10.2514/2.4231

Betts, J. T. (2010). Advances in design and control. Practical methods for optimal control and estimation using nonlinear programming (2nd ed.). Siam. https://doi.org/10.1137/1.9780898718577

Conway, B. A. (2012). A survey of methods available for the numerical optimization of continuous dynamic systems. Journal of Optimization Theory and Applications, 152(2), 271-306. https://doi.org/10.1007/s10957-011-9918-z

Fahroo, F., \& Ross, I. M. (2008). Advances in pseudospectral methods for optimal control. In AIAA Guidance, Navigation and Control Conference and Exhibit (pp. 1-23). Honolulu, Hawaii. https://doi.org/10.2514/6.2008-7309

Garg, D. (2011). Advances in global pseudospectral methods for optimal control [Doctoral dissertation, University of Florida].

Grimm, W., \& Hiltmann, P. (1987). Direct and indirect approach for real-time optimization of flight paths. In Optimal control (pp. 190-206). Springer. https://doi.org/10.1007/BFb0040209

Hargraves, C. R., \& Paris, S. W. (1987). Direct trajectory optimization using nonlinear programming and collocation. Journal of Guidance, Control, and Dynamics, 10(4), 338-342. https://doi.org/10.2514/3.20223

Huang, G., Lu, Y., \& Nan, Y. (2012). A survey of numerical algorithms for trajectory optimization of flight vehicles. Science China Technological Sciences, 55(9), 2538-2560.

https://doi.org/10.1007/s11431-012-4946-y

Jalali-Naini, S. H., \& Ebrahimi, M. (2017, May). Second-order optimal line-of-sight guidance law for minimum and nonminimum phase control systems. In 2017 International Conference on Mechanical, System and Control Engineering (ICMSC) (pp. 225-229). IEEE.

https://doi.org/10.1109/ICMSC.2017.7959476

Jalali-Naini, S. H., \& Sajjadi, S. H. (2016). First-order optimal line-of-sight guidance for stationary targets. Scientia Iranica. Transaction B, Mechanical Engineering, 23(2), 588-599. https://doi.org/10.24200/sci.2016.3846

Kamyar, R., \& Taheri, E. (2014). Aircraft optimal terrain/threatbased trajectory planning and control. Journal of Guidance, Control, and Dynamics, 37(2), 466-483.

https://doi.org/10.2514/1.61339
Kassaei, S. I., \& Kosari, A. (2018). Aircraft trajectory planning with an altitude-bound in terrain-following flight. Modares Mechanical Engineering, 17(12), 135-144.

Kazemifar, O., Babaei, A. R., \& Mortazavi, M. (2017). Online aircraft velocity and normal acceleration planning for rough terrain following. The Aeronautical Journal, 121(1244), 15611577. https://doi.org/10.1017/aer.2017.27

Kosari, A., \& Kassaei, S. I. (2019). TF/TA optimal flight trajectory planning using a novel regenerative flattener mapping method. Scientia Iranica. https://doi.org/10.24200/sci.2019.51314.2109

Kosari, A., Maghsoudi, H., \& Lavaei, A. (2017). Path generation for flying robots in mountainous regions. International Journal of Micro Air Vehicles, 9(1), 44-60.

https://doi.org/10.1177/1756829316678877

Kosari, A., Maghsoudi, H., Lavaei, A., \& Ahmadi, R. (2015). Optimal online trajectory generation for a flying robot for terrain following purposes using neural network. In Proceedings of the Institution of Mechanical Engineers, Part G: Journal of Aerospace Engineering, 229(6), 1124-1141. https://doi.org/10.1177/0954410014545797

Lindfield, G., \& Penny, J. (2018). Numerical methods: using MAT$L A B$ (4th ed.). Academic Press.

Malaek, S. M., \& Kosari, A. R. (2007). Novel minimum time trajectory planning in terrain following flights. IEEE Transactions on Aerospace and Electronic Systems, 43(1), 2-12. https://doi.org/10.1109/TAES.2007.357150

Malaek, S. M., \& Kosari, A. R. (2012). Dynamic based cost functions for TF/TA flights. IEEE Transactions on Aerospace and Electronic Systems, 48(1), 44-63. https://doi.org/10.1109/TAES.2012.6129620

Pourtakdoust, S. H., Kiani, M., \& Hassanpour, A. (2011). Optimal trajectory planning for flight through microburst wind shears. Aerospace Science and Technology, 15(7), 567-576. https://doi.org/10.1016/j.ast.2010.11.002

Rao, A. V. (2009). A survey of numerical methods for optimal control. Advances in the Astronautical Sciences, 135(1), 497-528.

Sharma, T. (2006). Optimum flight trajectories for terrain collision avoidance [Master thesis, RMIT University].

Sharma, T., Bil, C., \& Eberhard, A. (2005). Control system for optimal flight trajectories for terrain collision avoidance. In International Conference on Knowledge-Based and Intelligent Information and Engineering Systems (pp. 622-627). Springer. https://doi.org/10.1007/11552451_85

Von Stryk, O., \& Bulirsch, R. (1992). Direct and indirect methods for trajectory optimization. Annals of Operations Research, 37(1), 357-373. https://doi.org/10.1007/BF02071065 